\title{
Transcranial Doppler in hemoglobin SC disease
}

\author{
Camilo Vieira $^{1,2}$ | Carolina Nogueira Costa de Oliveira ${ }^{3}$ | Ludmila Araújo Borges de \\ Figueiredo $^{3}$ | Rayra Pereira Santiago ${ }^{2,4}$ | Corynne Stephanie Ahouefa Adanho ${ }^{2,4}$ | \\ Sanzio Silva Santana ${ }^{2,4}$ | Caroline Lang Burak ${ }^{5}$ | Isa Menezes Lyra ${ }^{5}$ | \\ Marilda Souza Goncalves 2,4
}

${ }^{1}$ Ambulatório Pediátrico de Doença Cerebrovascular, Hospital Universitário Professor Edgard Santos, Universidade Federal da Bahia, Bahia, Brazil

${ }^{2}$ Centro de Pesquisas Gonçalo Moniz, Fundação Oswaldo Cruz, Bahia, Brazil

${ }^{3}$ Universidade Salvador, Bahia, Brazil

${ }^{4}$ Departamento de Análises Clínicas e Toxicológicas, Faculdade de Farmácia, Universidade Federal da Bahia, Bahia, Brazil

${ }^{5}$ Departamento de Hematologia, Hospital Universitário Professor Edgard Santos, Universidade Federal da Bahia, Bahia, Brazil

Correspondence

Camilo Vieira, Pediatric Cerebrovascular Disease Outpatient Center, Hospital Universitario Professor Edgard Santos, Universidade Federal da Bahia, Rua Augusto Viana. s/n, Canela, Salvador, Bahia 40110-060, Brasil. Email:dr.camilovieira@gmail.com

\begin{abstract}
Background: Stroke is a severe clinical disorder in sickle cell disease (SCD), and few studies have evaluated transcranial Doppler (TCD) flow velocities in hemoglobin SC disease (HbSC). The guidelines for stroke risk are based on evaluations in sickle cell anemia (SCA) or $\mathrm{HbS} / \beta$ thalassemia.

Procedure: In this study, we compare cerebral blood flow in patients with SCD stratified by genotypes. A total of 1,664 pediatric patients with SCD underwent TCD velocity screening, and the time-averaged maximum mean velocity (TAMM) was determined in the middle cerebral artery (MCA), anterior cerebral artery (ACA), and distal intracranial internal carotid artery (ICA).

Results: Abnormal velocities were not identified in the ACA; therefore, we only use ICA and MCA velocities. TAMM from the left and right in the ICA and MCA was $134.3 \pm 32.0$ and $134.4 \pm 32.6$ $\mathrm{cm} / \mathrm{s}$ in patients with SCA, and $105.2 \pm 20.6$ and $104.7 \pm 20.0 \mathrm{~cm} / \mathrm{s}$ in the patients with $\mathrm{HbSC}$, respectively. Mean TAMM between right and left ICA/MCA was $134.5 \pm 30.5 \mathrm{~cm} / \mathrm{s}$ in the SCA group, and $104.9 \pm 19.3 \mathrm{~cm} / \mathrm{s}$ in the HbSC group. Notably, our data show that TCD velocities were significantly lower among the patients with $\mathrm{HbSC}$ compared to SCA. TAMM was negatively correlated with hemoglobin and hematocrit in both genotypes.

Conclusion: These results suggest that a different cut-off value for abnormal TCD velocities could be considered for patients with $\mathrm{HbSC}$. Additional studies are warranted to determine the actual risk of stroke in $\mathrm{HbSC}$ genotype associated with this possible TCD risk value.
\end{abstract}

KEYWORDS

hemoglobinopathies, neurology and sickle cell, sickle cell disease, transcranial Doppler ultrasound

\section{1 | INTRODUCTION}

Stroke is a common clinical manifestation in sickle cell disease (SCD) in children of 1 year or older. ${ }^{1-5}$ However, there are differences in stroke incidence among the SCD genotypes, with a rate of $0.61 / 100$ patients/year for sickle cell anemia (SCA) patients, $0.17 / 100$ patients/year for hemoglobin SC disease (HbSC), 0.11/100 patients/year for $\mathrm{HbS} / \beta^{+}$thalassemia, and $0.11 / 100$ patients/year for $\mathrm{HbS} / \beta^{0}$ thalassemia. $^{3}$

The transcranial Doppler (TCD) monitors the cerebral mean blood flow velocities of patients with SCD allowing the identification of

Abbreviations: ACA, anterior cerebral artery; HbSC, hemoglobin SC disease; ICA, Intracrania internal carotid artery; MCA, Middle cerebral artery; SCA, sickle cell anemia; SCD, sickle cell disease; SD, standard deviation; STOP, Stroke Prevention in Sickle Cell Anemia; TAMM,

time-averaged maximum mean velocity; TCD, transcranial Doppler those with an increased risk to developing stroke. ${ }^{6-10}$ The stratification of stroke risk can be determined by measuring the average maximum velocity or the time-averaged maximum mean velocity (TAMM) in the distal intracranial internal carotid artery (ICA), anterior cerebral artery (ACA), and middle cerebral artery (MCA). Values $\geq 200 \mathrm{~cm} / \mathrm{s}$ are considered of high risk, whereas values $<170 \mathrm{~cm} / \mathrm{s}$ are considered of low risk; speeds $\geq 170 \mathrm{~cm} / \mathrm{s}$ and $<200 \mathrm{~cm} / \mathrm{s}$ are considered conditional. ${ }^{6}$ After the first episode of stroke in SCA patients, there is a 46-90\% risk of stroke recurrence without prophylactic red cell transfusion therapy. ${ }^{11,12}$ Despite the high incidence of stroke in patients with $\mathrm{HbSC}$ compared with the pediatric population without SCD, 3,4 few studies have evaluated flow velocities by TCD in this patient subset, using TCD values for measuring risk stratification that are obtained from SCA or $\mathrm{HbS} / \beta$ thalassemia patients. 3,6,11 Therefore, theoretically, these values may not extrapolate well to patients with $\mathrm{HbSC}$. The aim 
of this study is to compare the characteristics of cerebral blood flow among patients with SCA and HbSC using TCD.

\section{2 | METHODS}

Patients with SCD evaluated from August 2011 to May 2015 in the Pediatric Cerebrovascular Disease Outpatient Center at the Hospital Universitario Professor Edgard Santos of the Universidade Federal da Bahia were included in the study. Only genotype HbSS and $\mathrm{HbSC}$ between 2 and 16 years old were included. Patients with a prior overt stroke event, on hydroxyurea therapy, with a simple transfusion in the last 3 months or on chronic blood therapy regimens were not included in the study. One examiner performed the TCD in all patients using the same device (Doppler; probe 2 Mhz model, Ezdop, Germany). The TAMM was determined in the ICA, ACA, and MCA. Since we did not identify abnormal values in the ACA, we only consider the highest velocity obtained of ICA and MCA. If TAMM in all arteries was between 70 and $170 \mathrm{~cm} / \mathrm{s}$, the examination was considered normal; the TAMM $\geq 170 \mathrm{~cm} / \mathrm{s}$, but less than $200 \mathrm{~cm} / \mathrm{s}$, in any one artery was considered conditional; the TAMM $\geq 200 \mathrm{~cm} / \mathrm{s}$ in an artery was considered abnormal, and the TAMM $<70 \mathrm{~cm} / \mathrm{s}$ was considered low. The failure to detect the flux wave during the examination was characterized as inadequate. Patients were analyzed and stratified according to SCD genotypes (SCA and $\mathrm{HbSC}$ ). Venous blood was collected from patients for hematological analysis using electronic cell counter Ruby Cell Dyn (Abbott Diagnostics, Lake Forest, IL).

The Shapiro-Wilk test was used to determine the quantitative variables distribution and the independent Student's t-test was used to compare the means among the groups of quantitative variables with normal distribution. The Spearman's rank correlation coefficient was used to measures the linear relation between quantitative data. The results were considered significant if the $P$ value was less than 0.05 . The data analysis was performed using SPSS version 21 (SPSS Inc., Chicago, IL).

This study was approved by the Research Board of the Secretary of Health of the state of Bahia (SESAB) 054/2011, and all parents or guardians provided written informed consent in accordance with the Helsinki Declaration of 1975 and its revision.

\section{3 | RESULTS}

A total of 2,774 patients with SCD were evaluated with TCD examination from August 2011 to April 2015 in the Pediatric Cerebrovascular Disease Center at the Hospital Universitario Professor Edgard Santos of the Universidade Federal da Bahia. A total of 1,110 patients were excluded (some had more than one exclusion criteria): standard deviation (SD) genotype: 10; sickle cell beta thalassemia: 70; age less than 2 years old or more than 16 years old: 75; prior overt stroke: 116 , blood transfusion therapy: 183; hydroxyurea use: 656 . A total of 1,664 SCD were investigated, with a mean \pm SD age of $6.5 \pm 3.8$ years, and $48.6 \%$ were females; 1106 (66.5\%) patients with SCA were investigated, with

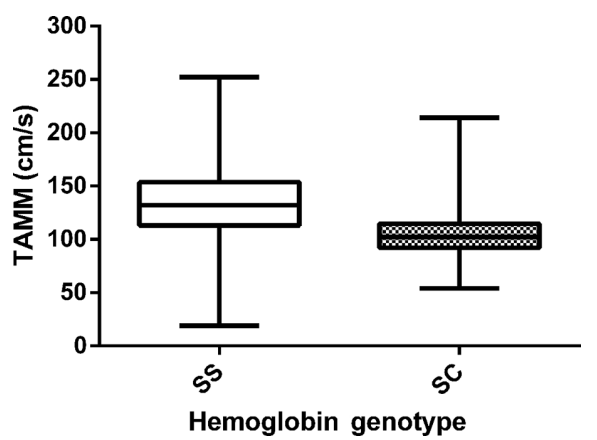

FIGURE 1 Time-averaged maximum mean velocity in the internal carotid artery and middle cerebral artery (ICA/MCA) in children and adolescents with sickle cell disease ( $\mathrm{HbSS}$ and $\mathrm{HbSC})(P<0.0001$; independent Student's t-test)

a mean age of $6.8 \pm 3.9$, and $47 \%$ were females. In addition, 558 (33.5 \%) patients with $\mathrm{HbSC}$ were investigated, with a mean age of $6.0 \pm 3.5$ years, and $51.6 \%$ were females.

The mean TAMM was $124.5 \pm 31.8$ and $124.4 \pm 32.2 \mathrm{~cm} / \mathrm{s}$ in the right and left ICA/MCA, respectively. The mean TAMM in the left and right ICA/MCA was $134.3 \pm 32.0$ and $134.4 \pm 32.6 \mathrm{~cm} / \mathrm{s}$ in the patients with SCA, and $105.2 \pm 20.6$ and $104.7 \pm 20.0 \mathrm{~cm} / \mathrm{s}$ in the patients with $\mathrm{HbSC}$, respectively. A mean TAMM between right and left ICA/MCA was $134.5 \pm 30.5 \mathrm{~cm} / \mathrm{s}$ in the SCA group, and $104.9 \pm 19.3 \mathrm{~cm} / \mathrm{s}$ in the HbSC group (Fig. 1). These differences were statistically significant $(P<0.001)$.

Hemoglobin and hematocrit were assessed in a smaller number of these patients. We evaluated 68 patients with $\mathrm{HbSC}$ and 79 patients with $\mathrm{HbSS}$. The TAMM was correlated with hemoglobin and hematocrit in both genotypes. In the HbSC genotype, a negative correlation was found between TAMM and hemoglobin $(R=-0.3390, P=0.007)$, and between TAMM and hematocrit $(R=-0.3470, P=0.0057)$ (Figs. 2A and 2B). In the HbSS genotype, a negative correlation was also found between TAMM and hemoglobin $(R=-0.2310, P=0.0447)$ and between TAMM and hematocrit $(R=-0.2649, P=0.0208)$ (Figs. 2C and 2D).

\section{4 | DISCUSSION}

A few studies have evaluated TCD examination of a large number of patients with SCD. ${ }^{13,14}$ Adams et al. ${ }^{13}$ published the TCD results from the Stroke Prevention in Sickle Cell Anemia (STOP) study, which was a clinical trial that included 5613 SCD children; they found that $67 \%$ of patients with SCD exhibited normal TCD results, $17.6 \%$ exhibited conditional TCD results, 9.3\% exhibited abnormal results, and $6.1 \%$ exhibited inadequate TCD evaluation. Another important study was performed by Enninful-Eghan et al., ${ }^{14}$ which evaluated the occurrence of stroke and the response to transfusion therapy in 475 patients with SCD over 8 years of follow-up prior to TCD examination, and in 530 patients with SCD over 8 years of follow-up with TCD. However, after some loss, the analysis of 404 patients with SCD revealed $14.4 \%$ with conditional TCD, $12.5 \%$ with abnormal, and $0.7 \%$ with an inconclusive 

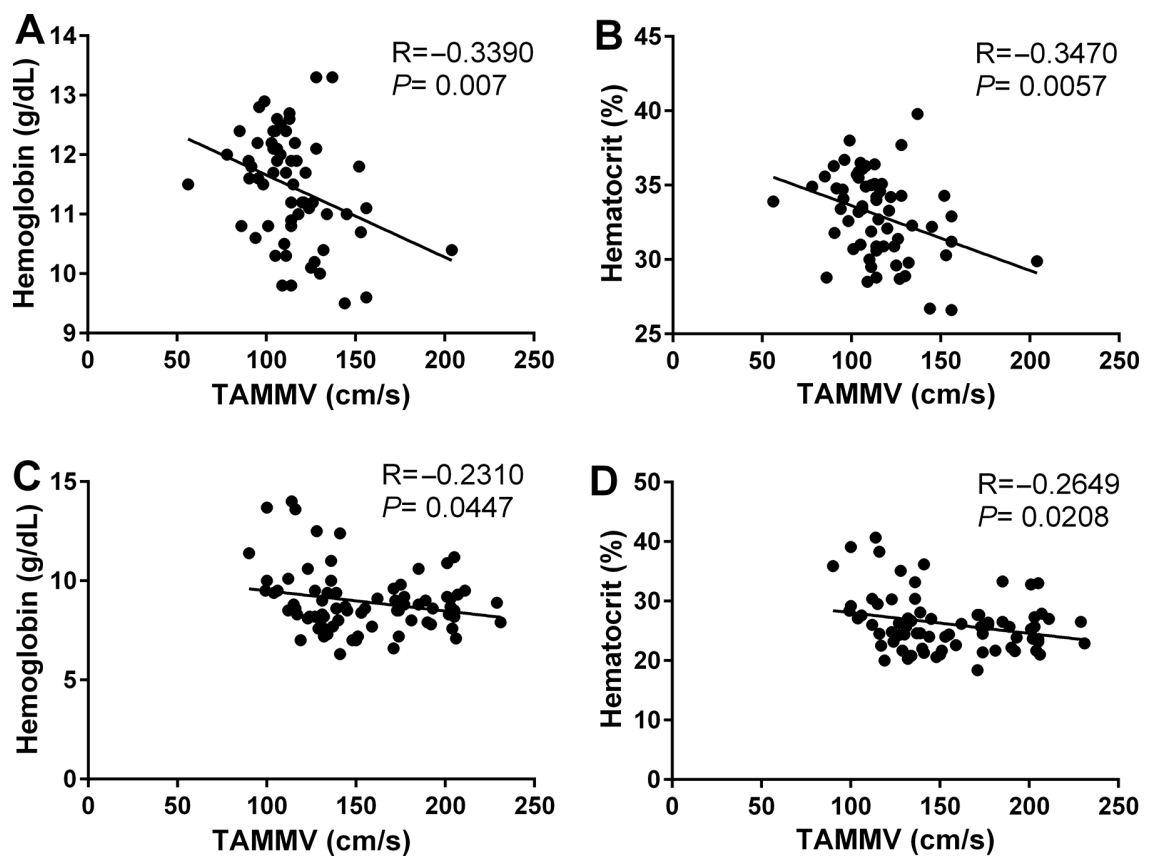

FIGURE 2 Correlations of maximum TAMM with markers of severity in patients with HbSS and HbSC. (A) Correlation between hemoglobin and TAMM in HbSC patients; (B) correlation between hematocrit and TAMM in HbSC patients; (C) correlation between hemoglobin and TAMM in HbSS patients; (D) correlation between hematocrit and TAMM in HbSS patients

TAB LE 1 Differences in transcranial Doppler (TCD) ultrasound screening among sickle cell anemia (HbSS) and sickle cell SC disease (HbSC)

\begin{tabular}{|c|c|c|c|c|c|c|}
\hline \multicolumn{7}{|c|}{ Transcranial Doppler result, N (\%) } \\
\hline Genotype & Normal, N (\%) & Conditional, $\mathrm{N}(\%)$ & Abnormal, $\mathrm{N}(\%)$ & Inconclusive, $\mathrm{N}(\%)$ & Low, $N(\%)$ & Total \\
\hline $\mathrm{HbSS}$ & $832(75.2)$ & $158(14.3)$ & $80(7.2)$ & $19(1.7)$ & $17(1.5)$ & 1,106 \\
\hline $\mathrm{HbSC}$ & $536(96.1)$ & $5(0.9)$ & $4(0.7)$ & $6(1.1)$ & $7(1.3)$ & 558 \\
\hline Total & 1,368 (82.2) & $163(9.8)$ & $84(5.0)$ & $25(1.5)$ & $24(1.4)$ & $1,664(100)$ \\
\hline
\end{tabular}

TCD evaluation. Additionally, the use of blood transfusion was successful in lowering TCD velocity among the patients with SCD, which was also demonstrated by Kwiatkowski et al. ${ }^{15}$

Our results differed from a previous report with 85 Brazilian children and teenagers with SCD, which found lower numbers of abnormal TCD. ${ }^{16}$ However, the difference between these two studies may be explained by the sample size, once our study evaluated a larger number of patients with SCD.

Deane et al. ${ }^{17}$ evaluated $47 \mathrm{TCD}$ tests from patients with $\mathrm{HbSC}$ and showed a TAMM velocity in the MCA of $94 \mathrm{~cm} / \mathrm{s}$. In our study, patients with $\mathrm{HbSC}$ had an average TAMM velocity of $104.9 \pm 19.3 \mathrm{~cm} / \mathrm{s}$ in the MCA/ICA. Rees's study did not identify individuals with high speeds according to the STOP protocol. ${ }^{6}$ This study analyzed a greater number of children and teenagers with $\mathrm{HbSC}$, and the comparison of patients with SCA showed that the TAMM velocities in patients with $\mathrm{HbSC}$ were significantly lower, in accord to previous report from our group. ${ }^{18}$ Using the current TCD velocity definitions of stroke risk for SCA, only $0.7 \%$ of patients with $\mathrm{HbSC}$ presented a high risk of stroke (Table 1). However, the differences in the mean TAMM velocities in the both MCA/ICA of SCA $(134.5 \pm 30.5 \mathrm{~cm} / \mathrm{s})$ and patients with $\mathrm{HbSC}$ $(104.9 \pm 19.3 \mathrm{~cm} / \mathrm{s})$ may suggest that different cut-off values should be used to define abnormal cerebral artery blood flow velocity and stroke risk for this genotype.

The average TAMM velocity in the MCA/ICA in patients with $\mathrm{HbSC}$ was $104.9 \mathrm{~cm} / \mathrm{s}$, with a SD of $19.3 \mathrm{~cm} / \mathrm{s}$, which allows us to consider a normal rate (two standard deviations) in patients with $\mathrm{HbSC}$ values above $143.5 \mathrm{~cm} / \mathrm{s}$. In this case, velocities $>143.5 \mathrm{~cm} / \mathrm{s}$ could be considered as a cut-off point for patients with HbSC. According to this parameter, 39 (7.0\%) individuals in our study would have high values. In the study conducted by Deane et al., ${ }^{17}$ the TAMM velocity in the 98th percentile was $128 \mathrm{~cm} / \mathrm{s}$ and the authors could not assign stroke risk to this population.

We found that TAMM was negatively correlated with hemoglobin and hematocrit in both genotypes. In a previous report, the increase in cerebral blood flow and flow velocity are associated with chronic anemia. ${ }^{6}$ These disorders can lead to cerebrovascular damage and stroke. ${ }^{6}$

In the present study, less than $1.6 \%$ of patients with $\mathrm{HbSC}$ presented TAMM higher than $170 \mathrm{~cm} / \mathrm{s}$. However, the mean MCA/ICA velocities were different between SCA and HbSC. A TAMM higher than $143.5 \mathrm{~cm} / \mathrm{s}$ can be considered as abnormal, but whether or not it means an increase in stroke risk is unknown. Follow-up brain 
MRI/MRA imaging, serial TCD exams, and close clinical monitoring would be needed to determine the actual risk of stroke for patients with $\mathrm{HbSC}$ with TAMM in this range.

\section{ACKNOWLEDGMENT}

We thank the nurses and all of the health professionals who provided technical support and assisted the sickle cell patients.

\section{CONFLICT OF INTEREST}

The authors declare that there is no conflict of interest.

\section{REFERENCES}

1. Ingram VM. A specific chemical difference between the globins of normal human and sickle-cell anaemia haemoglobin. Nature. 1956;178(4537):792-794.

2. Steinberg MH, Rodgers GP. Pathophysiology of sickle cell disease: Role of cellular and genetic modifiers. Semin Hematol. 2001;38(4):299-306.

3. Ohene-Frempong K, Weiner SJ, Sleeper LA, et al. Cerebrovascular accidents in sickle cell disease: Rates and risk factors. Blood. 1998;91(1):288-294.

4. Broderick JP, Brott TG, Duldner JE, Tomsick T, Huster G. Volume of intracerebral hemorrhage. A powerful and easy-to-use predictor of 30-day mortality. Stroke. 1993;24(7):987-993.

5. Adams RJ. TCD in sickle cell disease: An important and useful test. Pediatr Radiol. 2005;35(3):229-234

6. Adams RJ, McKie VC, Hsu L, et al. Prevention of a first stroke by transfusions in children with sickle cell anemia and abnormal results on transcranial Doppler ultrasonography. New Engl J Med. 1998;339(1):5-11.

7. Adams RJ, McKie VC, Carl EM, et al. Long-term stroke risk in children with sickle cell disease screened with transcranial Doppler. Ann Neurol. 1997;42(5):699-704.

8. Adams RJ, Nichols FT, Figueroa R, McKie V, Lott T. Transcranial Doppler correlation with cerebral angiography in sickle cell disease. Stroke. 1992;23(8):1073-1077.

9. Sloan MA, Alexandrov AV, Tegeler $\mathrm{CH}$, et al. Assessment: Transcranial Doppler ultrasonography: Report of the Therapeutics and Technology Assessment Subcommittee of the American Academy of Neurology. Neurology. 2004;62(9):1468-1481.
10. Eicke BM, Tegeler CH, Dalley G, Myers LG. Angle correction in transcranial Doppler sonography. J Neuroimag. 1994;4(1):29-33.

11. Hankins JS, Fortner GL, McCarville MB, et al. The natural history of conditional transcranial Doppler flow velocities in children with sickle cell anaemia. Br J Haematol. 2008;142(1):94-99.

12. Brambilla DJ, Miller ST, Adams RJ. Intra-individual variation in blood flow velocities in cerebral arteries of children with sickle cell disease. Pediatr Blood Cancer. 2007;49(3):318-322.

13. Adams RJ, Brambilla DJ, Granger S, et al. Stroke and conversion to high risk in children screened with transcranial Doppler ultrasound during the STOP study. Blood. 2004;103(10):3689-3694.

14. Enninful-Eghan H, Moore RH, Ichord R, Smith-Whitley K, Kwiatkowski JL. Transcranial Doppler ultrasonography and prophylactic transfusion program is effective in preventing overt stroke in children with sickle cell disease. J Pediatr. 2010;157(3):479-484.

15. Kwiatkowski JL, Yim E, Miller S, Adams RJ. Effect of transfusion therapy on transcranial Doppler ultrasonography velocities in children with sickle cell disease. Pediatr Blood Cancer. 2011;56(5):777-782.

16. Hokazono M, Silva GS, Silva EM, Braga JA. Results from transcranial Doppler examination on children and adolescents with sickle cell disease and correlation between the time-averaged maximum mean velocity and hematological characteristics: A cross-sectional analytical study. Sao Paulo Med J. 2011;129(3):134-138.

17. Deane CR, Goss D, O'Driscoll S, et al. Transcranial Doppler scanning and the assessment of stroke risk in children with HbSC [corrected] disease. Arch Dis Child. 2008;93(2):138-141.

18. Vieira CO, Oliveira CNC, Figueiredo L, Burak C, Lyra IM, MS G. Doppler transcraniano na doença falciforme: Qual o valor de referência para crianças e adolescentes com genótipo SC? Braz J Hematol Hemother. 2014;36:1.

How to cite this article: Vieira Camilo, de Oliveira Carolina Nogueira Costa, de Figueiredo Ludmila Araújo Borges, Santiago Rayra Pereira, Adanho Corynne Stephanie Ahouefa, Santana Sanzio Silva, Burak Caroline Lang, Lyra Isa Menezes, Goncalves Marilda Souza (2016). Transcranial Doppler in hemoglobin SC disease. Pediatric Blood \& Cancer, XX, XX-XX. DOI: 10.1111/pbc.26342. 